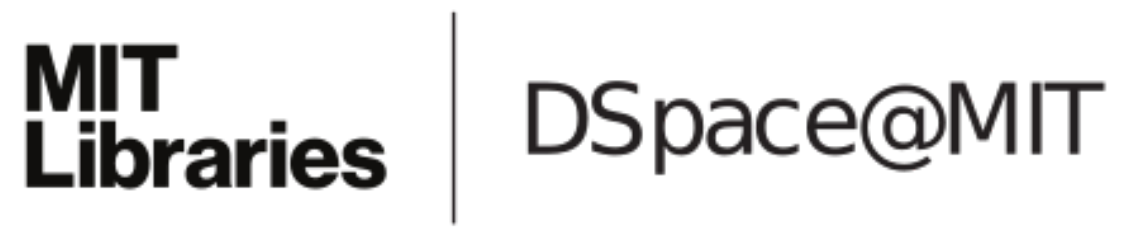

\author{
MIT Open Access Articles
}

Concurrencer: a tool for retrofitting concurrency into sequential Java applications via concurrent libraries

The MIT Faculty has made this article openly available. Please share how this access benefits you. Your story matters.

Citation: Dig, Danny, Marrero, John J., and Ernst, Michael D. (2009). Concurrencer: a tool for retrofitting concurrency into sequential Java applications via concurrent libraries. 31st International Conference on Software Engineering companion volume, 2009 (Piscataway, N.J.: IEEE): 399-400. ( 2009 IEEE

As Published: http://dx.doi.org/10.1109/ICSE-COMPANION.2009.5071031

Publisher: Institute of Electrical and Electronics Engineers

Persistent URL: http://hdl.handle.net/1721.1/58989

Version: Final published version: final published article, as it appeared in a journal, conference proceedings, or other formally published context

Terms of Use: Article is made available in accordance with the publisher's policy and may be subject to US copyright law. Please refer to the publisher's site for terms of use. 


\title{
Concurrencer: a Tool for Retrofitting Concurrency into Sequential Java Applications via Concurrent Libraries
}

\author{
Danny Dig, John Marrero, Michael D. Ernst \\ Massachusetts Institute of Technology \\ Computer Science and Artificial Intelligence Laboratory \\ \{dannydig,marrero,mernst $\} @$ csail.mit.edu
}

\begin{abstract}
Parallelizing existing sequential programs to run efficiently on multicores is hard. The Java 5 package java.util. concurrent (j.u.c.) supports writing concurrent programs. To use this package, programmers still need to refactor existing code. This is tedious, error-prone, and omission-prone.

This demo presents our tool, CONCURRENCER, which enables programmers to refactor sequential code into parallel code that uses j.u.c. concurrent utilities. CONCURRENCER does not require any program annotations, although the transformations span several, non-adjacent, program statements and use custom program analysis. A find-and-replace tool can not perform such transformations. Empirical evaluation shows that CONCURRENCER refactors code effectively: CONCURRENCER correctly identifies and applies transformations that some open-source developers overlooked, and the converted code exhibits good speedup.
\end{abstract}

\section{Introduction}

The computing hardware industry has shifted to multicore processors. This demands that programmers find and exploit parallelism in their programs, if they want to reap the same performance benefits as in the past.

The dominant paradigm for concurrency in desktop programs is shared-memory, thread-based. However, this paradigm increases the risk for deadlocks and data-races, commonly known as thread-safety concerns. In addition, the programmer needs to consider scalability concerns as well: will the parallelized program continue to run faster when adding more parallel resources?

To meet the needs of programmers with respect to thread-safety and scalability, the Java standard library has been extended with a package, java.util.concurrent (from here on referred as j.u.c.), containing several util- ity classes for dealing with concurrency. Among others, j.u.c. contains a set of Atomic classes which offer thread-safe, lock-free programming over single variables, and several thread-safe abstract data types (e.g., ConcurrentHashMap) optimized for scalability. Java 7 will contain a framework Fork/Join Task ${ }^{1}$ for finegrained parallelism of intensive computations.

However, manually refactoring a program to use $j \cdot u . c$. utilities is tedious because it requires changing many lines of code, is error-prone because programmers can use the wrong APIs, and is omission-prone because programmers can miss opportunities to use the enhanced APIs.

This demo presents Concurrencer, our extension to Eclipse's refactoring engine. CONCURRENCER enables Java programmers to quickly and safely refactor their sequential programs to use j.u.c. utilities. In this demo we present three refactorings: (i) CONVERT INT to ATOMICINTEGer, (ii) CONvert hashMap to Concurrenthashmap, and (iii) Convert RecurSION TO FJTASK.

The first two refactorings are "enabling transformations", i.e., they make a program thread-safe, but do not introduce multi-threading into a single-threaded program. Our previous study [1] of five open-source projects that were manually parallelized by their developers shows that these two refactorings were among some of the most commonly used in practice. The third refactoring introduces multi-threading: it converts a sequential recursive divideand-conquer algorithm into one which solves the subproblems in parallel using ForkJoinTasks.

For evaluation, we compared the manually refactored code in 6 open-source projects with code refactored automatically. The results show that ConCURRENCER is effective and the parallel code exhibits good speedup.

A more detailed description of ConcurRENCER can be found in the ICSE'09 research track [2]. CONCURRENCER can be downloaded from: http://refactoring.info/tools/Concurrencer

\footnotetext{
${ }^{1}$ http://gee.oswego.edu/dl/concurrency-interest/
} 


\section{Concurrencer}

Supported Refactorings. The first refactoring, ConVERT INT to ATOMicInTEger, enables a programmer to convert an int field to an AtomicInteger. AtomicInteger is a lock-free utility class which encapsulates an int value and provides update operations that execute atomically. Our refactoring replaces field updates with calls to AtomicInteger's APIs.

For example, a common update pattern on an int field is (i) read the current value, (ii) add delta, and (iii) update the field value. To make this update thread-safe, the three operations need to execute atomically. Traditionally, programmers use locks to ensure atomicity. Due to the program having to frequently acquire and release the lock, the program does not scale well under heavy lockcontention. Concurrencer finds such read/add/update code patterns and replaces them with a call to Atomic Integer's getAndAdd () which atomically executes the update without locks (instead it uses efficient compare-and-swap).

The second refactoring, Convert HashMap to ConcurrenTHASHMAP, enables a programmer to convert an HashMap field to ConcurrentHashMap, a thread-safe, highly scalable implementation for hash maps. Our refactoring replaces map update patterns with calls to Concurrent HashMap's atomic APIs.

For example, a common update pattern is (i) check if a map contains a $\langle k e y$, value $\rangle$ pair, and if it is not present, (ii) place the pair in the map. For thread-safety, the two operations need to execute atomically. Traditionally, a programmer would use a map-common lock. Since all accesses to the map have to acquire the map's lock, this can severely degrade the map's performance. CONCURRENCER replaces such an updating pattern with a call to ConcurrentHashMap's put IfAbsent which atomically executes the update without locking the entire map.

The third refactoring, Convert Recursion to FJTAsk, converts a sequential divide-and-conquer algorithm into an algorithm which solves the recursive subproblems in parallel using the Fork/Join Task framework. Our refactoring encapsulates the subproblems as ForkJoinTasks and passes them to the framework for effective scheduling.

For example, a sequential mergesort (array) first checks whether the input array is of trivial size (and sorts it directly), otherwise splits the array into two halves, sorts each half, and then merges the sorted halves. CONCURREnCER parallelizes this algorithm using the skeleton of the sequential algorithm. For the base-case it (i) checks whether the array is smaller than a user-defined threshold and (ii) invokes the original sequential sort. For the recursive case, it creates ForkJoinTasks for each of the two halves, schedules the two tasks in parallel, waits for the computations to finish, and then merges the two sorted halves.
Implementation. CONCURRENCER is implemented as an extension to Eclipse's refactoring engine, thus it conveniently provides previewing the code changes, undo, etc. The programmer only needs select the method or field to be refactored, and the concurrency refactoring (and the sequential threshold in case of CONVERT RECURSION TO FJTASK).

CONCURRENCER's program analysis determines (i) whether it is safe to remove synchronization locks that might protect field accesses, (ii) its data-flow analysis determines what variables are written in the update patterns and read afterward, and assigns them appropriately when the update pattern is replaced with a single API call. The analysis and the code edits are implemented on top of Eclipse's JDT.

Evaluation. We used ConCurrencer to refactor the same fields that the open-source developers of Tomcat, MINA, JaxLib, Zimbra, GlassFish, and Struts refactored to AtomicInteger or Concurrent HashMap. There were a total of 141 such refactorings. Using Concurrencer, the developers could have saved changing 1019 LOC manually.

We then compared the manually vs. automatically refactored programs. In terms of errors in using the j.u.c. APIs, the open source developers 4 times erroneously replaced infix expressions like $++f$ with $f$.getAndIncrement () (which corresponds to the postfix expression $\mathrm{f}++$ ). CONCURRENCER used the correct API calls. In terms of missing opportunities to convert from old update patterns to the new atomic APIs, the programmers missed 43 out of 83 such opportunities. CONCURRENCER only missed 10 opportunities.

We also used Concurrencer to parallelize 6 divide-andconquer algorithms using CONVERT ReCURSION to FJTASK. CONCURRENCER changed 302 LOC and the parallelized code exhibits on average $1.84 x$ speedup on a 2 -core machine and $3.28 x$ speedup on a 4-core machine.

\section{Conclusions}

Refactoring sequential code to concurrency is not trivial. Even seemingly simple refactorings like replacing data types with thread-safe, scalable implementations provided in java.util. concurrent, is prone to human errors and omissions. This demo presents CONCURRENCER which automates three refactorings. Our preliminary experience with CONCURRENCER shows that it is more effective than a human developer in identifying and applying such transformations.

\section{References}

[1] D. Dig, J. Marrero, and M. D. Ernst. How Do Programs Become More Concurrent? A Story of Program Transformations. Tech Report MIT-CSAIL-TR2008-053.

[2] D. Dig, J. Marrero, and M. D. Ernst. Refactoring Sequential Java Code for Concurrency via Concurrent Libraries. To Appear in Proceedings of ICSE'09. 